\title{
The influence of magnetic fields on the hydrostatic structure of the atmospheres of chemically peculiar stars
}

\author{
G. Valyavin ${ }^{1,2}$, O. Kochukhov ${ }^{3}$, and N. Piskunov ${ }^{4}$ \\ 1 Special Astrophysical Observatory, Russian Academy of Sciences, Nizhnii Arkhyz, \\ Karachai Cherkess Republic 369167, Russia \\ 2 Isaac Newton Institute of Chile, SAO Branch, Russia \\ 3 Institute for Astronomy, University of Vienna, Türkenschanzstraße 17, 1180 Vienna, Austria \\ e-mail: kochukhov@astro.univie.ac.at \\ ${ }^{4}$ Uppsala Astronomical Observatory, Box 515, 75120 Uppsala, Sweden \\ e-mail: piskunov@astro.uu.se
}

Received 18 September 2003 / Accepted 4 March 2004

\begin{abstract}
We discuss the properties of atmospheres of chemically peculiar magnetic stars. The slow evolution of global magnetic fields leads to the development of an induced electric current in all conductive atmospheric layers. The Lorentz force, which results from the interaction between a magnetic field and the induced current, may change the atmospheric structure and provide insight into the formation and evolution of stellar magnetic fields. We developed a model atmosphere code that takes into account the Lorentz force in the equation of hydrostatic equilibrium, and computed a number of model atmospheres for magnetic A and B stars. The surface distribution of a magnetic field was assumed to be a dipole, slightly distorted by the induced atmospheric electric current. The interaction between the magnetic field and electric current is modelled in detail, taking into account microscopic properties of the stellar plasma. The presence of a significant Lorentz force leads to substantial modification of the atmospheric structure and in particular the pressure stratification, which in turn influences the formation of absorption spectral features, especially hydrogen Balmer lines. Furthermore, we find that rotational modulation of the diskaverage parameters of a global stellar magnetic field causes characteristic rotational variability of hydrogen lines. With our model, observable effects correspond to induced electric currents of the order of $10^{-11} \mathrm{cgs}$, which requires characteristic field evolution times 2-3 orders of magnitude shorter than the field decay time estimated for magnetic A and B stars assuming fossil dipolar field topology in the stellar interior. Using the computations of our model atmospheres we consider an observational aspect of the problem and attempt to interpret photometric data on the variability of hydrogen lines within the framework of simplest model of the evolution of global magnetic fields. With the hydrogen line data we find tentative support for the presence of a non-negligible Lorentz force in the atmospheres of some magnetic chemically peculiar stars.
\end{abstract}

Key words. stars: chemically peculiar - stars: magnetic fields - stars: atmospheres

\section{Introduction}

Magnetic chemically peculiar (CP) stars comprise $10-15 \%$ of the main sequence B-F stars and are distinguished by slow rotation, large overabundances of rare earth and some iron-peak elements as well as by the presence of strong global magnetic fields on their surfaces. Statistical properties of the fields in CP stars and results of detailed modelling of field geometries in individual objects are generally consistent with the picture of smooth, quasi-dipolar or quadrupolar magnetic field, inclined with respect to the stellar axis of rotation (e.g. Landstreet 2001; Bagnulo et al. 2002).

Despite a common acknowledgment of the profound effect that magnetic fields may have on the photospheric structure of peculiar stars, they have been analyzed using mainly standard

Send offprint requests to: G. Valyavin, e-mail: gendoz@sao.ru plane-parallel non-magnetic models atmospheres. Calculation of the synthetic Stokes parameter spectra based on such models allows mapping the surface magnetic fields and determination of other fundamental stellar parameters. The general properties (effective temperatures, surface gravities, magnetic field strengths, etc.) of the majority of magnetic stars have been investigated in such a way. However, many important questions, connected with the nature of magnetic fields in hot stars, cannot be addressed in this manner. The most important of these are associated with the problem of the origin and evolution of global stellar magnetic fields, which remains among the few long-standing mysteries of stellar astrophysics.

It is understood that any variation of the global stellar magnetic field (related, for instance, to a global field decay) leads to development of the induced electric currents in conductive atmospheric layers. The Lorentz force, which appears as a 
result of the interaction between the magnetic field and electric currents, may noticeably affect the atmospheric structure and influence the formation of spectral lines. The strength and the sign of the Lorentz force depend on the geometry of the surface magnetic field and on the electric field induced by secular evolution of the magnetic field. Techniques of mapping magnetic field topology in hot stars are well-developed and available experimental data, in particular high-resolution spectropolarimetric observations, are sufficient to reconstruct fine details of the magnetic structures on the surfaces of CP stars (e.g., Bagnulo et al. 2002; Kochukhov et al. 2002, 2004). On the other hand, the current geometry is impossible to study directly, as it is determined by a combination of various complex processes of field formation and evolution acting in subphotospheric layers and in stellar interiors. At the moment theoretical understanding and the corresponding computational methods applied to study magnetic fields of hot stars are inadequate for detailed modelling of the field evolution using only a small number of well-justified basic assumptions (Moss 2001). Therefore, an empirical determination of the strength and orientation of the Lorentz force in stellar atmospheres can supply information about the magnitude and structure of electric currents and hence provide crucial insights into the physics of stellar magnetism.

For example, if the magnetic field of a typical CP star is a fossil and approximately dipolar, as suggested by Borra \& Landstreet (1980) and Borra et al. (1982), and its characteristic decay time is about $10^{10} \mathrm{yr}$ (Moss 1984), then the field is nearly force-free (i.e., the Lorentz force is close to zero) and does not significantly change the hydrostatic structure of stellar atmospheres (Landstreet 1987). In reality, these theoretical predictions may be questionable, since some of the observational data on CP stars obtained in the last twenty years present evidence for the existence of non-force-free magnetic fields in stellar photospheres. Furthermore, some recent statistical studies of the evolutionary state of the magnetic CP stars (Glagolevskij \& Chountonov 2001; Hubrig et al. 2000) propose that the characteristic time of the evolution of global stellar magnetic fields may be considerably shorter than predicted in earlier theoretical analyses.

If these conclusion are correct, then the concept of essentially dipolar, very slowly decaying fossil magnetic fields in deep interiors of CP stars must be revised. From this point of view, the Lorentz force appears to be the unique observable which can be employed for investigations of the field behaviour inside a magnetic star. Furthermore, taking it into account may be necessary in regular model atmosphere and spectrum synthesis calculations for magnetic CP stars.

The next section reviews the most important papers, connected with the atmospheric analysis of the effects of nonforce-free magnetic fields for the middle main sequence stars. In the following (Sect. 3) we formulate the scope and targets of the present investigation. Section 4 introduces the theoretical background that we use, the basic equations and the assumptions necessary for modelling. In Sect. 5 we describe the method of model atmosphere calculations and give details of the model atmosphere code that we employ. Section 6 describes the results of our calculations. Observational aspects of the problem and general discussion are presented in Sects. 7 and 8 respectively.

\section{Historical background}

The first self-consistent atmospheric calculations, which included the effects due to the global magnetic field, were presented by Stępień (1978). He considered an inward and outward directed magnetic force as a term in the equation of hydrostatic equilibrium. It was assumed that the Lorentz force in stellar atmospheres arises from the distortion of a decentered dipolar field and the distribution of the atmospheric electric currents is determined by the requirement that the atmosphere of a magnetic star remains in static equilibrium. Stępien showed that the inclusion of the magnetic force in model atmosphere calculations can produce observable changes in the absorption lines of neutral hydrogen.

In the 1980s there were several attempts to determine the Lorentz force in the atmospheres of magnetic stars by analysis of the wings of hydrogen Balmer lines (see, for instance, Madej 1981, 1983a,b; Madej et al. 1984; Musielok \& Madej 1988). In this series of papers the authors presented results of the photometric search for rotational variability of the $\mathrm{H} \beta$ line in the spectra of magnetic CP stars. It was found that a number of studied magnetic stars indeed show variations of $\mathrm{H} \beta$ strength with the rotational period, and this is a rather typical phenomenon for magnetic CP stars. The authors also concluded that the electromagnetic forces produce a significant contribution to the observed Balmer line variations. In an attempt to model these effects Madej (1981, 1983a) adopted the following model. The total magnetic field of a star is a slightly distorted oblique dipole. This distortion is equivalent to the presence of small azimuthal electric currents $j_{\phi}$ of the assumed factorized form

$j_{\phi}=j(r) \sin \theta$,

vanishing at the magnetic poles $\left(\theta=0^{\circ}, 180^{\circ}\right)$ and outside the atmosphere. The atmosphere is affected by the Lorentz force, entering the equation of hydrostatic equilibrium. If the strength of the atmospheric electric current (and thus the Lorentz force) is large enough to change the atmospheric structure considerably, it also changes some absorption features observed in stellar spectra (in particular hydrogen Balmer lines affected by linear Stark broadening). For a given set of conditions the effect of the Lorentz force produced by a dipolar field is maximal at the magnetic equator and vanishes at the magnetic pole. Therefore, rotating magnetic stars are expected to show Balmer line variations with the same period as the changes of light and magnetic field strength, well known for CP stars.

Similar models were considered in other studies (e.g. Carpenter 1985). The authors usually discussed the influence of the magnetic force on the atmospheric plasma and modelled the Lorentz force resulting from the interaction between a dipolelike total field and a small additional magnetic field caused by a weak electric current appearing due to the distortion of the dipole symmetry (Stępień 1978; Madej 1983a). They derived the distribution of this current from the boundary conditions and the assumption of static equilibrium of the model. This 
approach is quite successful in describing general effects of the geometrical distortion of stellar shape due to the presence of a non-force-free magnetic field. However, the method has some weaknesses. For example, one of the main problems is that models developed in previous studies did not include the microscopic equations describing the interaction between magnetic field and electric current. As a result, these models were unable to properly describe the distribution of the Lorentz force in the vertical direction in stellar atmospheres.

Another aspect of the influence of the magnetic field on stellar atmospheres was discussed by Landstreet (1987). He criticized the static equilibrium approach of Stępień (1978) and suggested one consider the Lorentz force induced by the decay of magnetic fields in slowly rotating CP stars. Assuming essentially a poloidal interior field, Landstreet concluded that its slow decay (with the predicted characteristic decay time of the order of $10^{10} \mathrm{yr}$ ) does not create any significant Lorentz force which could change the atmospheric structure considerably except at small optical depths. Despite this result, Landstreet noted that such a large characteristic decay time can be considered only as an upper limit. In most real stars the global field decay combined with other dynamical processes leads to the development of additional plasma flows (such as meridional circulation) that can make the decay time shorter by a factor of 20 or more. In addition, the global field structure in deep interiors of real stars may be quite different from poloidal due to the effects of, for example, differential rotation and the presence of a convective core. All this may reduce the predicted decay time of global stellar fields by orders of magnitude and result in a significant influence of the Lorentz force on the atmospheric structure.

In general, consideration of any global magnetic topology gives us a large collection of possibilities for generating surface electric currents even without invoking the global magnetic field evolution. There are mechanisms that may produce significant atmospheric currents even in constant magnetic fields. For instance, Peterson \& Theys (1981) considered the interaction of the horizontal component of a magnetic field with a flow of charged particles drifting in the atmosphere under the influence of the radiation pressure. The result of such an interaction is that drifting particles acquire some horizontal velocity component. This leads to the appearance of the Lorentz force which may be significant for the hottest known magnetic stars. More recently LeBlanc et al. (1994) studied a similar physical situation in the context of ambipolar diffusion.

It is clear that the problem of the influence of magnetic field on the hydrostatic structure of stellar atmospheres deserves careful investigation. The studies mentioned above suggested that magnetic forces may lead to some differences between the atmospheric structures of magnetic and non-magnetic stars. This conclusion has important implications for the atmospheric analysis of the magnetic stars. As it follows from the discussion given by Stępień (1978), Madej (1983a,b), Carpenter (1985) and LeBlanc et al. (1994), the Lorentz force may noticeably change the effective gravity and may influence the formation of pressure-sensitive spectral features, especially hydrogen Balmer lines. Some of the photometric data presented by Madej et al. (1984) and Musielok \& Madej (1988) can be considered as an evidence for the presence of non-force-free magnetic fields. Spectroscopy of hydrogen lines also points in this direction. For example, Kroll (1989) found variability with amplitude of more than $1 \%$ of the Balmer lines in magnetic stars. He showed that at least part of this variability can be attributed to the presence of a non-zero Lorentz force in the atmospheres of magnetic stars.

In spite of this observational progress, there is no unambiguous answer to the question of whether or not a consideration of non-force-free magnetic fields is important in atmospheric modelling. What fundamental knowledge can we obtain from a study of non-force-free configurations of stellar magnetic fields? Thus, the question of the importance of the Lorentz force in the construction of model atmospheres of magnetic CP stars is still open.

\section{The scope of the present study}

The problem of how the Lorentz force influences the atmospheric structure has been discussed by a number of authors, but, in our opinion, this question deserves additional study. The main difficulty of the previous theoretical investigations is that their examination of the Lorentz force in the magnetic CP stars required knowledge of the exact geometry of the global magnetic field and electric currents. We propose an alternative approach to tackle this problem and, following the pioneering works by Stępień, Madej, Carpenter and Landstreet, we developed a new, more detailed model for the stellar hydrostatic equilibrium with the Lorentz force included.

Our method is based on a semi-empirical consideration of the problem. We concede that our knowledge of the processes in stellar interiors and available computational methods are inadequate for a fully self-consistent derivation of the field geometry and the structure of the induced electric currents through a computation of realistic time-dependent 3D stellar magnetohydrodynamical models. Instead, we adopt an axisymmetric loworder multipolar geometry of the surface field, which is known to give a reasonable approximation for the majority of CP stars, and focus our investigation on a refined quantitative analysis of the local stellar magnetohydrostatic equilibrium. We vary the sign and amplitude of the Lorentz force in model atmosphere calculations and investigate the resulting perturbations in the atmospheric structure and emergent stellar spectra. We demonstrate that, if the Lorentz force is sufficiently strong to make these perturbations observable, the sign and magnitude of the Lorentz force can be measured directly, independently of its nature. Such measurements are equivalent to mapping the induced atmospheric electric current and, together with detailed information about magnetic field geometry, provide the foundation for major advances in our understanding of the fundamental physics of magnetic stars.

In contrast to the approaches favoured by the authors of previous studies, our model is based on the detailed consideration of microscopic electromagnetic properties of atmospheric currents in the presence of a magnetic field. This has made it possible to take into account the effects of the partial ionisation and magnetoresistivity (the reduction of electric conductivity in the presence of a magnetic field), which becomes especially 
important in the upper atmospheric layers where the cyclotron frequency of the current particles is higher than the effective collisional frequency.

Although detailed analysis of the nature of the generated atmospheric currents lies beyond the scope of our study, in Sect. 8 we discuss the possibility that a non-zero Lorentz force is related to the actual temporal changes in the structure of the global magnetic field. Using a schematic model of the field decay we apply our calculations to interpret photometric data on the variability of hydrogen lines available in the literature and estimate typical time scales of the evolution of global magnetic fields in CP stars.

\section{The theory and basic assumptions}

The equation of hydrostatic equilibrium, which includes the Lorentz force term, has the form

$\nabla P=\rho g+f_{\mathrm{L}}$,

$f_{\mathrm{L}}=\frac{1}{c} j \times \boldsymbol{B}$

per unit volume. Using Ampere's law,

$\boldsymbol{j}=\frac{c}{4 \pi} \nabla \times \boldsymbol{B}$,

the Lorentz force can be written as

$\boldsymbol{f}_{\mathrm{L}}=\frac{1}{4 \pi}(\nabla \times \boldsymbol{B}) \times \boldsymbol{B}=-\frac{\nabla \boldsymbol{B}^{2}}{8 \pi}+\frac{1}{4 \pi}(\boldsymbol{B} \cdot \nabla) \cdot \boldsymbol{B}$,

or in tensor notations

$\left(f_{\mathrm{L}}\right)_{i}=\frac{\partial}{\partial x_{j}}\left(-\delta_{i j} \frac{B^{2}}{8 \pi}+\frac{B_{i} B_{j}}{4 \pi}\right)$.

As one can see, the magnetic field $\boldsymbol{B}$ creates in the plasma an isotropic pressure $\frac{B^{2}}{8 \pi}$ and tension $\frac{B_{i} B_{j}}{4 \pi}$ directed along the magnetic lines of force. While neighbouring lines of force of a magnetic field try to expand due to the magnetic pressure, tension tends to compensate for this effect. The force-free configuration is possible only when the gradient of the magnetic pressure is fully compensated by the tension forces

$\frac{\nabla \boldsymbol{B}^{2}}{8 \pi}=\frac{1}{4 \pi}(\boldsymbol{B} \cdot \nabla) \cdot \boldsymbol{B}$.

It is evident from Eq. (4) that any magnetic geometry for which $\nabla \times \boldsymbol{B}=0$ or $\nabla \times \boldsymbol{B}=h(\boldsymbol{r}) \boldsymbol{B}$ (where $h(\boldsymbol{r})$ is a scalar function) corresponds to the force-free configuration. The classical example of such a field is an axisymmetric magnetic dipole (see also Parker 1979, Sect. 5.1). To acquire a non-zero force, a dipolar magnetic configuration needs to be distorted or decentered. It is clear that idealized examples of highly symmetric magnetic field geometries like a dipole do not exist. Even in the case of a slowly rotating star with an initially dipolar force-free magnetic field the global field decay leads to the development of induced atmospheric currents and as a result, additional components of the magnetic field appear and lead to distortions of the ideal force-free geometry of the dipolar field. The strength of the generated electric currents is not large, but even in this idealized case the Lorentz force due to the interaction between magnetic field and induced electric current may have an influence on the upper atmospheric layers. In all other more realistic situations, typical of magnetic stars across the HR diagram, the Lorentz forces cannot be ignored in the atmospheric calculations. For instance, the cool stars with an external convective envelope have very complex magnetic fields concentrated in magnetic flux tubes with a field strength of a few kG. Such fields are very far from being force-free and we do not discuss them in this paper. The main goal of our study is to examine the slowly rotating CP stars that are believed to have surface magnetic fields of low-order multipolar topology. We consider atmospheric electric currents induced by the evolution of the global magnetic field. Other scenarios of the generation of the surface currents, such as ambipolar diffusion, are ignored. As it follows from the discussion given by Landstreet (1987), our model should be rather close to reality for slowly rotating main sequence stars in a wide temperature interval from 8000 to $20000 \mathrm{~K}$.

\subsection{The model assumptions}

We consider a model similar to the model examined by Madej (1983a): the surface magnetic field has a configuration of a nearly axisymmetric low-order multipolar field. As an example of such a magnetic topology we examine a dipolar field, but note that considerations presented below can be easily extended to any axisymmetric poloidal configuration. The magnetic field in the deep stellar interior may have a configuration quite different from the surface magnetic topology and does not explicitly enter in our analysis.

To calculate the hydrostatic structure accounting for the magnetic force term we introduce the following basic assumptions:

1. the magnetized atmospheric layers under consideration are assumed to be in static equilibrium;

2. the stellar surface magnetic field is dominated by the dipolar force-free component $(\nabla \times \boldsymbol{B}=0)$ in all atmospheric layers. The vector components of a dipolar field in spherical coordinates in the reference frame of the dipole are given by

$$
\begin{aligned}
& B_{r}=\frac{R_{\star}^{3} B_{\mathrm{p}}}{r^{3}} \cos \theta, \\
& B_{\theta}=\frac{R_{\star}^{3} B_{\mathrm{p}}}{2 r^{3}} \sin \theta, \\
& B_{\phi}=0,
\end{aligned}
$$

where $B_{\mathrm{p}}$ is the polar magnetic field strength, $\theta$ and $\phi$ are magnetic co-latitude and azimuth respectively, and $r$ and $R_{\star}$ correspond to the radial coordinate and stellar radius;

3. distortion of the dipolar field is created by the field evolution and can be presented through the induced azimuthal electric current $\boldsymbol{j}$. Such a distortion is equivalent to the additional magnetic field $\boldsymbol{B}_{\text {add }}$, which follows from the Maxwell equation,

$\nabla \times \boldsymbol{B}_{\mathrm{add}}=\frac{4 \pi}{c} \boldsymbol{j}$ 
4. deviations of the distribution of induced electric currents from axial symmetry caused by dynamical effects related to the stellar rotation and possible presence of Hall's current are neglected.

We start by considering the equation of the pressure balance which has the form

$\nabla \boldsymbol{P}_{\mathrm{tot}}=\rho \boldsymbol{g}+\frac{1}{c} \boldsymbol{j} \times \boldsymbol{B}$,

where $\boldsymbol{P}_{\text {tot }}$ is the total atmospheric pressure (gas + turbulence + radiation), $\rho$ is the gas density, $\boldsymbol{g}$ is the gravitational acceleration, $\boldsymbol{j}$ denotes the induced atmospheric electric current and $\boldsymbol{B}$ the magnetic field vector.

In the present paper we do not describe the Lorentz force through a detailed study of the geometry and long-term evolution of the additional magnetic field $\boldsymbol{B}_{\text {add }}$ over the whole stellar surface. Instead, we directly specify a plausible form of the atmospheric electric current, generated by such field evolution, and use its amplitude and orientation as effective free parameters to define the magnetic force term in the equation of hydrostatic equilibrium.

Due to the axial symmetry of the dipolar field, its evolution creates an electric current with only an azimuthal component: $j_{\phi}=j_{\mathrm{eq}} \sin \theta$, where $j_{\mathrm{eq}}$ is an atmospheric current at the equatorial plane of the dipole. In this case the scalar radial component of Eq. (10) has the following form:

$\frac{\partial P_{\mathrm{tot}}}{\partial r}=-\rho g \pm \frac{j_{\mathrm{eq}}}{c} B_{\theta} \sin \theta$,

where the positive sign refers to the case of an outward directed Lorentz force and the negative sign is for an inward directed magnetic force. This formula can be extended to any axisymmetric poloidal field by taking into account that, in general, the surface distribution of induced currents can be expressed with Legendre polynomials $j_{\phi} \sim P_{n}^{1}(\cos \theta)$ (Wrubel 1952), where $n=1$ for dipole, $n=2$ for quadrupole, etc.

For the dipolar field the electric current $j_{\mathrm{eq}}$ induced at the equatorial plane can be described by the function

$j_{\mathrm{eq}}=\Phi\left(\lambda, E_{\mathrm{eq}}, B_{\mathrm{eq}}\right)$

Here $\lambda$ is the electrical conductivity, $E_{\mathrm{eq}}$ is the equatorial surface electric field induced by the magnetic field evolution and $B_{\mathrm{eq}}$ denotes the equatorial strength of a magnetic field. Now we will discuss this equation in more detail.

\subsection{Properties of the atmospheric electric current in the presence of a magnetic field}

In the general case, Ohm's law in the presence of a magnetic field has the following approximate form (Sect. 2 in Pikelner 1966; Cowling 1957; Schluter 1950):

$\boldsymbol{j}=\lambda \boldsymbol{E}_{\|}+\lambda_{1} \boldsymbol{E}_{\perp}-\frac{\lambda_{2}}{B} \boldsymbol{E}_{\perp} \times \boldsymbol{B}$,

where $\boldsymbol{E}_{\|}$and $\boldsymbol{E}_{\perp}$ are electric field components directed, respectively, along and across the magnetic field lines, $\lambda$ is the electrical conductivity in the absence a magnetic field, $\lambda_{1}$ is the conductivity across the magnetic field lines and $\lambda_{2}$ is the Hall's conductivity. As follows from the inspection of the properties of a self-gravitating plasma (Pikelner 1966; Cowling 1957; Schluter 1950), the electrical conductivity across the magnetic field lines $\lambda_{1}$ is reduced in comparison to the non-magnetic conductivity $\lambda$ :

$\lambda_{1}=\frac{\lambda}{1+(\omega \tau)^{2}}$.

Here $\omega$ and $\tau$ are the cyclotron frequency and the mean freepath time of the conducting particles. Hall's current (the third term on the right-hand side of Eq. (13)) appears due to the drift of charged particles spiralling in a magnetic field. The direction of Hall's current is defined by the vector multiplication $\boldsymbol{E}_{\perp} \times \boldsymbol{B}$. At the equatorial plane of a dipolar field, for example, Hall's current is radially directed. The electrical conductivity of such a current is defined by the expression

$\lambda_{2}=\frac{\lambda \omega \tau}{1+(\omega \tau)^{2}}$.

As one can see from this equation, assumption 3 introduced above may be violated. In general, the presence of a magnetic field leads to distortion of a simple symmetrical distribution of an atmospheric current. In our case, for example, the assumption that the electric current can be described only through the azimuthal component becomes questionable. In addition, the presence of the drifting components of an atmospheric electric current may lead to additional induced electric fields and development of global plasma drift motions similar to meridional circulation (cf. Landstreet 1987). These effects can only be studied with self-consistent 3D time-dependent magnetohydrodynamical calculations, which are outside the scope of our present investigation. To make the problem tractable in the context of our study and to make it possible to incorporate the effects of the Lorentz force in standard 1D plane-parallel stellar model atmospheres we disregard Hall's currents in our calculations. We note that deviations from an idealized situation mainly take place in the upper atmospheric layers where the cyclotron frequency of the charged particles is higher than the effective collisional frequency. In this section we will carry out a quantitative assessment to characterize the possible deviation of a magnetic field in our model from the equilibrium configuration. This discussion is also relevant for models suggested in previous studies, since these models were also affected by the same deviation from an equilibrium situation.

As follows from Eqs. (13)-(15), Hall's current is negligible in the deepest atmospheric layers where $\omega \tau \ll 1$. Note that in these layers the electrical conductivity across the magnetic field lines is close to the non-magnetic case. Now let us define a formal condition when Hall's current cannot be neglected. The atmospheric layer where

$\omega \tau=1$

corresponds to the situation where the electrical conductivity across the field lines is reduced relative to the non-magnetic one by a factor of two. On the other hand, this layer also defines the point in the atmosphere (where $\lambda_{2} \approx \lambda_{1} \approx \frac{\lambda}{2}$ ) above which Hall's current increases rapidly. Thus, we choose Eq. (16) as a 


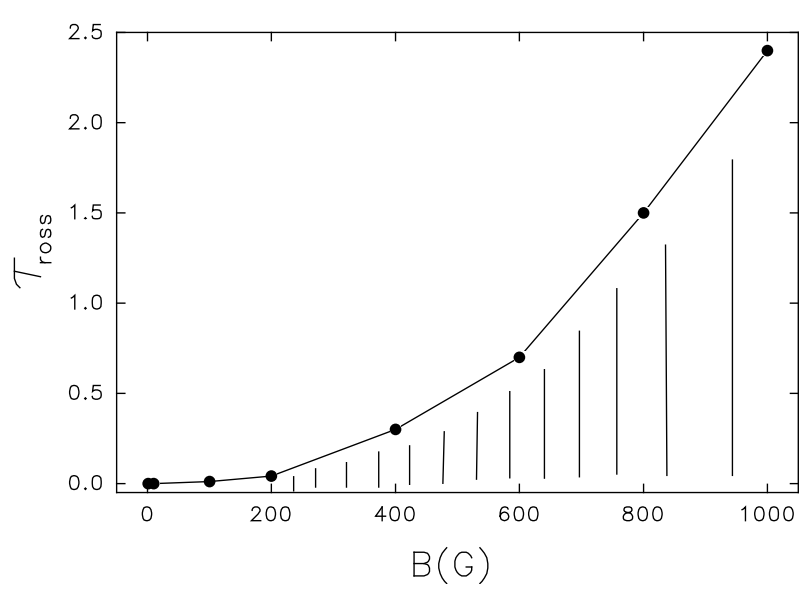

Fig. 1. The dependence of the critical optical depth $\tau_{\text {ross }}$ from the magnetic field strength.

criterion for determining the region of validity of our assumptions.

Using a standard non-magnetic Kurucz (1993) model atmosphere of a star with $T_{\text {eff }}=11000 \mathrm{~K}, \log g=4.0$ and solar chemical composition we estimated the relation between the magnetic field strength and the critical Rosseland optical depth $\left(\tau_{\text {ross }}\right)$ defined by Eq. (16). This relation is presented in Fig. 1. The dashed area under the curve corresponds to the atmospheric layers for which the component of Hall's current due to electrons cannot be ignored. As one can see from this figure, neglecting of Hall's current is strictly adequate only for weak-field ( $B \lesssim 0.5 \mathrm{kG}$ ) magnetic stars. Nevertheless, even when Hall's current becomes important, it increases the electric conductivity across the magnetic field lines, but, to a first approximation, does not produce a radially directed force which would make a significant contribution to the hydrostatic equilibrium equation. We note that with our model it will be possible to verify the role of the Hall's current observationally, by using rotational variability of the spectroscopic features formed at small optical depths (for instance, the cores of the Balmer lines), where effects due to Hall's current are expected to be maximal.

In the situation when the relative importance of the Hall effect remains uncertain, we consider the whole range of typical stellar magnetic field strengths. This will allow us to make the most general conclusions about the effects due to magnetic field and to investigate the likely lower limit of the modification of atmospheric structure that one can expect to find in magnetic CP stars.

Now we can rewrite Ohm's law as follows:

$\boldsymbol{j}=\lambda_{1} \boldsymbol{E}_{\perp}$

Note that due to our assumptions, the component of the induced electric current $\boldsymbol{E}_{\|}$parallel to the magnetic field is absent in the model. Thus, the final working expression for the induced electric current has the following scalar form:

$j_{\phi}=\frac{E_{\mathrm{eq}} \lambda}{1+(\omega \tau)^{2}} \sin \theta$
Generalizing our approach to all kinds of atmospheric charged particles we present our solution for the radial component of the Lorentz force through the effective gravity

$\frac{\partial P_{\mathrm{tot}}}{\partial r}=-\rho g_{\mathrm{eff}}$,

$g_{\mathrm{eff}}=g \pm \sum_{i} \frac{\lambda_{i} \sin \theta}{c \rho\left(1+\left(\omega_{i} \tau_{i}\right)^{2}\right)} E_{\mathrm{eq}} B_{\theta}$,

where the index $i$ runs through all types of charged particles. The method for evaluation of the electrical conductivity in the absence of magnetic field is detailed in the Appendix.

Since nothing can be said about the equatorial electric field $E_{\text {eq }}$ generated by the evolution of the global stellar field, we will attempt to develop a method that will allow one to determine this value from observations. Our further considerations are based on the assumption that $E_{\text {eq }}$ can, as a first approximation, be treated as a free parameter. Empirical determination of this quantity will make it possible to select realistic models of the interior magnetic field and test them in the context of magnetic field evolution. We will return to this problem in Sect. 8.

\section{Model atmosphere calculations}

We selected the program ATLAS9 (Kurucz 1993) as the basis for our model atmosphere calculations. The following additional assumptions specific to our computations with this model atmosphere code were adopted.

1. The local model atmosphere is represented by planeparallel layers;

2. opacity sources are calculated as non-magnetic;

3. convection is neglected and radiative equilibrium persists throughout the whole stellar atmosphere;

4. local thermodynamic equilibrium is assumed;

5. the chemical composition of the atmosphere is solar.

For the solution of the equation of hydrostatic equilibrium we developed new subroutines that calculate the pressure balance for horizontally oriented magnetic field lines. To obtain the input model atmosphere for the modification of hydrostatic equilibrium due to magnetic forces, our modified ATLAS9 code calculates the standard non-magnetic model. After evaluating concentrations and electrical conductivity of all gas species, a new model is calculated including a magnetic force term. Iterative corrections to the temperature and other local atmospheric parameters are repeated until convergence is achieved.

In the next stage, to be able to model real observational data, we need to build a model representative of the whole star. The most general way to achieve this is to

i) calculate (for given $E_{\mathrm{eq}}$ and the polar strength $B_{\mathrm{p}}$ of the assumed dipolar magnetic topology) a number of local model atmospheres and synthetic spectra for all surface areas from the magnetic equator to the pole;

ii) integrate the local spectra over the visible stellar hemisphere for a set of rotational phases and given orientation of the stellar rotational and magnetic axes with respect to the line-of-sight. 
Table 1. Model atmospheres computed for different magnetic field strengths.

\begin{tabular}{cc|cc}
\hline \hline $\begin{array}{l}\text { Model } \\
\text { No. }\end{array}$ & $\begin{array}{c}B_{\text {eq }} \\
(\mathrm{G})\end{array}$ & $\begin{array}{l}\text { Model } \\
\text { No. }\end{array}$ & $\begin{array}{c}B_{\text {eq }} \\
(\mathrm{G})\end{array}$ \\
\hline 1 & 3 & 10 & 500 \\
2 & 5 & 11 & 750 \\
3 & 10 & 12 & 1000 \\
4 & 25 & 13 & 3000 \\
5 & 50 & 14 & 6000 \\
6 & 100 & 15 & 12000 \\
7 & 200 & 16 & 18000 \\
8 & 300 & 17 & 25000 \\
9 & 400 & 18 & 50000 \\
\hline
\end{tabular}

Additional study revealed that even the most extreme magnitude of the Lorentz force that we tested did not change the limb darkening of a magnetic model compared to a nonmagnetic one. Thus, for this first investigation of the properties of magnetic atmospheres we used a simplified approach and simulated disk-integrated stellar characteristics with a single magnetic model calculated for the disk-averaged magnetic parameter $\left\langle\sin \theta B_{\theta}\right\rangle$. This approximate method is described in Sect. 6.2 and assumes a linear response of the properties of magnetic atmospheres to changes in the input parameters.

\section{Numerical results}

\subsection{Local magnetic atmosphere at the equatorial plane}

We start with discussion of the modification of the stellar atmosphere at the magnetic equator. The induced electric field is constant with depth and has only an azimuthal component $E_{\phi}=E_{\text {eq }}$. Our calculations showed that for magnetic field strengths of several hundred gauss a scalar value of $E_{\text {eq }} \cdot B_{\text {eq }}$ of about $10^{-9}-10^{-10}$ (cgs units) corresponds to the case when the influence of the magnetic field induces considerable changes in the atmospheric structure. We first examine the outwardand inward-directed Lorentz force at the magnetic equator in a star with effective temperature $T_{\text {eff }}=11000 \mathrm{~K}$, surface gravity $\log g=4.0$ and $E_{\text {eq }}=5 \times 10^{-11}$ (hereafter we quote $E_{\text {eq }}$ in cgs units of $\mathrm{cm}^{-1 / 2} \mathrm{~g}^{1 / 2} \mathrm{~s}^{-1}$ ). First we analyse the effective gravity, $g_{\text {eff }}$, in comparison with the reference non-magnetic model. Figure 2 shows behaviour of $g_{\text {eff }}$ with the Rosseland optical depth for a number of chosen models. The different curves in this figure correspond to models listed in Table 1. As expected, the effective gravity is decreased (increased) relative to the nonmagnetic model in the case of the outward (inward) directed Lorentz force in a wide range of optical depths. This wellknown result follows from the fact that a significant magnetic force term in the equation of hydrostatic equilibrium decreases (for the case of the outward-directed Lorentz force) or increases (for the inward-directed Lorentz force) the gas pressure of the magnetized stellar atmosphere. Interestingly enough, we find that the behaviour of the effective gravity shows two extrema. This can be understood with the help of careful consideration of the microscopic processes taking place in our model.
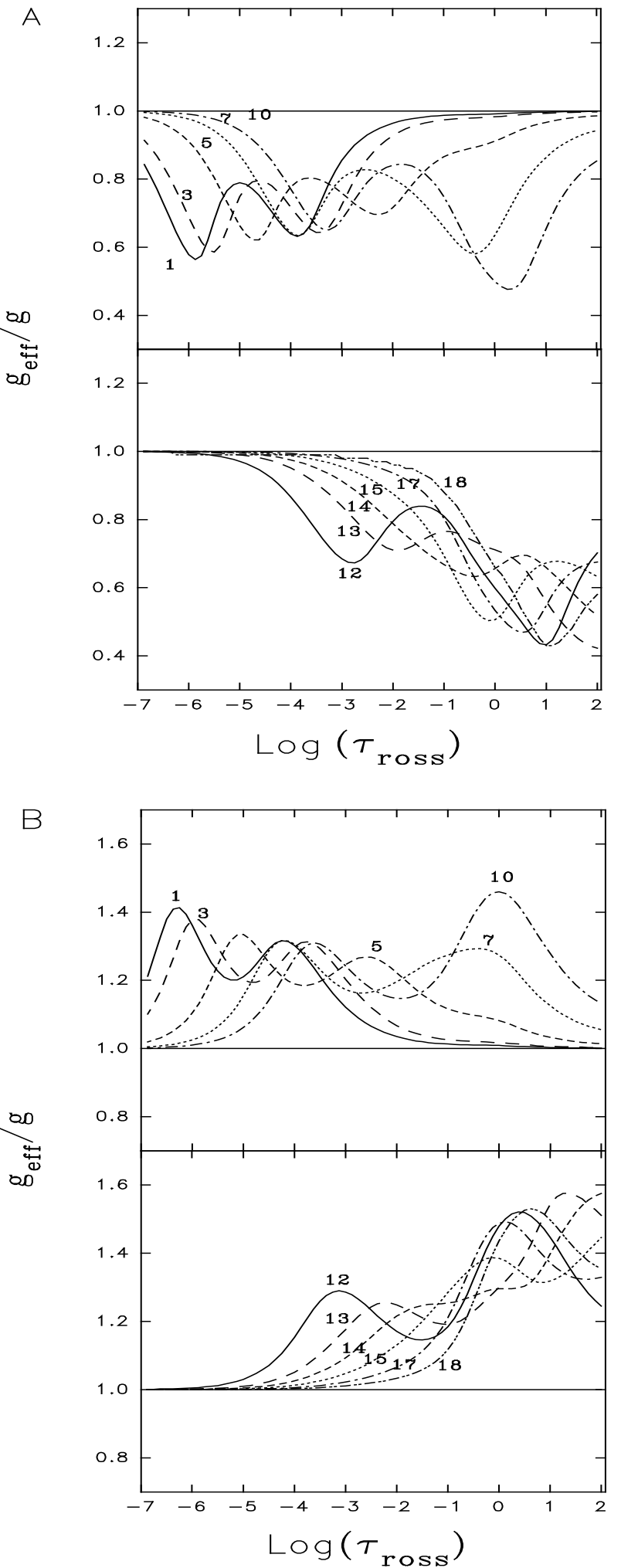

Fig. 2. The effective gravity versus the Rosseland optical depth for a) the outward- and b) inward-directed Lorentz force.

As one can see from Eq. (20), for a given vector multiplication $\boldsymbol{E} \times \boldsymbol{B}$ (constant with depth), the radial behaviour of the effective gravity depends only on the competition of the electrical conductivity and the gas density. The electrical conductivity of each gas component in the direction perpendicular 
to the magnetic field lines is given by Eq. (14). In the most dense layers the total electrical conductivity is the same as in the non-magnetic case and is defined mainly by free electrons. The location of the first strong gravity disturbance in the deepest atmospheric layers, where $\omega_{\mathrm{e}} \tau_{\mathrm{e}} \ll 1$, is explained by the fact that with decreasing depth the gas density falls faster than the electronic conductivity. This situation and corresponding gravity anomaly continue up to the layers where $\omega_{\mathrm{e}} \tau_{\mathrm{e}} \sim 1$. In the upper atmospheric layers the electronic conductivity goes quickly to zero and the effective gravity shifts back to the original gravitational acceleration.

The second extremum in the $g_{\text {eff }} / g$ curve is similar to the first one, but this time the gravity anomaly is defined by the ion component of the electrical conductivity. This is illustrated in Fig. 3, where electrical conductivity of electrons and ions is plotted as a function of the Rosseland optical depth for models Nos. 5 and 10. This figure reflects the well-known fact that electric currents in the non-magnetic plasma mainly consist of electrons, i.e. the electronic conductivity is larger by a few orders of magnitude than the ionic conductivity, which can, therefore, be ignored in a wide range of calculations of non-magnetic plasma. However, the effect of magnetoresistivity in magnetized plasma leads to the situation where detailed calculation of the ionic conductivity becomes important. Electrons are light and have a high cyclotron frequency. Thus, the magnetic field decreases the electronic conductivity more effectively than the conductivity due to heavy ions. There are regions in the magnetized stellar atmospheres where the electronic conductivity is decreased almost to zero and the atmospheric current is supported only by ions. As one can see from Fig. 3, this happens in the outer atmospheric layers, where the cyclotron frequency of electrons is higher than the collisional frequency, but the cyclotron frequency of ions is still less than the collisional frequency.

Thus, within the framework of our model, a bimodal structure in the behaviour of effective gravity appears due to the competition between the electronic and ionic conductivities. In the uppermost atmospheric layers (where $\omega_{\mathrm{i}} \tau_{\mathrm{i}} \gg 1, \lambda$ ) the magnetoresistivity ensures that the stellar atmosphere is close to the force-free configuration. However, this conclusion can be modified by the presence of the diffusional components of electric current, such as Hall's current, which can be significant in the uppermost layers, but are ignored in our model.

The strengths of the stellar magnetic field and the equatorial electric field mainly influence the depth of characteristic perturbations of the effective gravity, but not its magnitude. Thus, even relatively weak magnetic fields can noticeably affect the structure of a magnetized atmosphere and the formation of certain spectral lines provided that the equatorial electric field is large enough.

Figure 4 shows the temperature structure of the magnetic models in comparison with the reference non-magnetic model. In addition to changing the effective gravity, the Lorentz force affects other atmospheric parameters. However, the main effect leading to the changes in emergent stellar radiation is the modification of the pressure-temperature relation, which can reach up to a factor of $2-3$. Since the temperature perturbations are small (below 1\%, see Fig. 4), the $P-T$ relation is
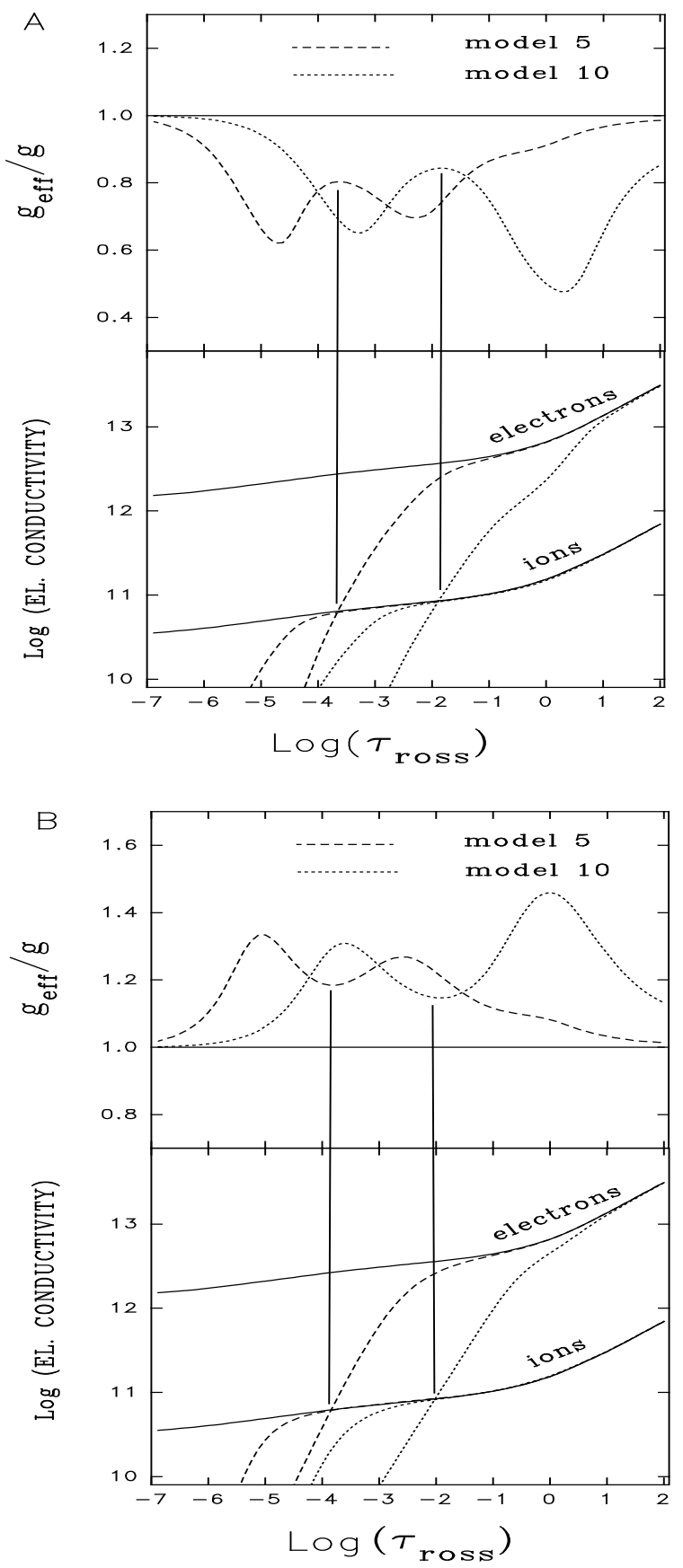

Fig. 3. The electron and ion electrical conductivity (logarithmic scale) as a function of the Rosseland optical depth for models No. 5 ( $B_{\mathrm{eq}}=$ $50 \mathrm{G})$ and $10\left(B_{\mathrm{eq}}=500 \mathrm{G}\right)$ (dashed curves). The electrical conductivity of the electrons and ions in the absence of magnetic field is shown by solid lines. a) The outward-directed Lorentz force, b) the inwarddirected magnetic force.

changed by the strong perturbation in the concentrations of the atmospheric particles. In the case of the outward-directed Lorentz force the concentration is decreased in a magnetized star. In other words, the atmosphere expands. If the Lorentz force is inward-directed, the atmosphere has a higher density and smaller scale hight.

Due to their high pressure sensitivity, hydrogen Balmer lines provide the best spectroscopic diagnostics of the stellar 

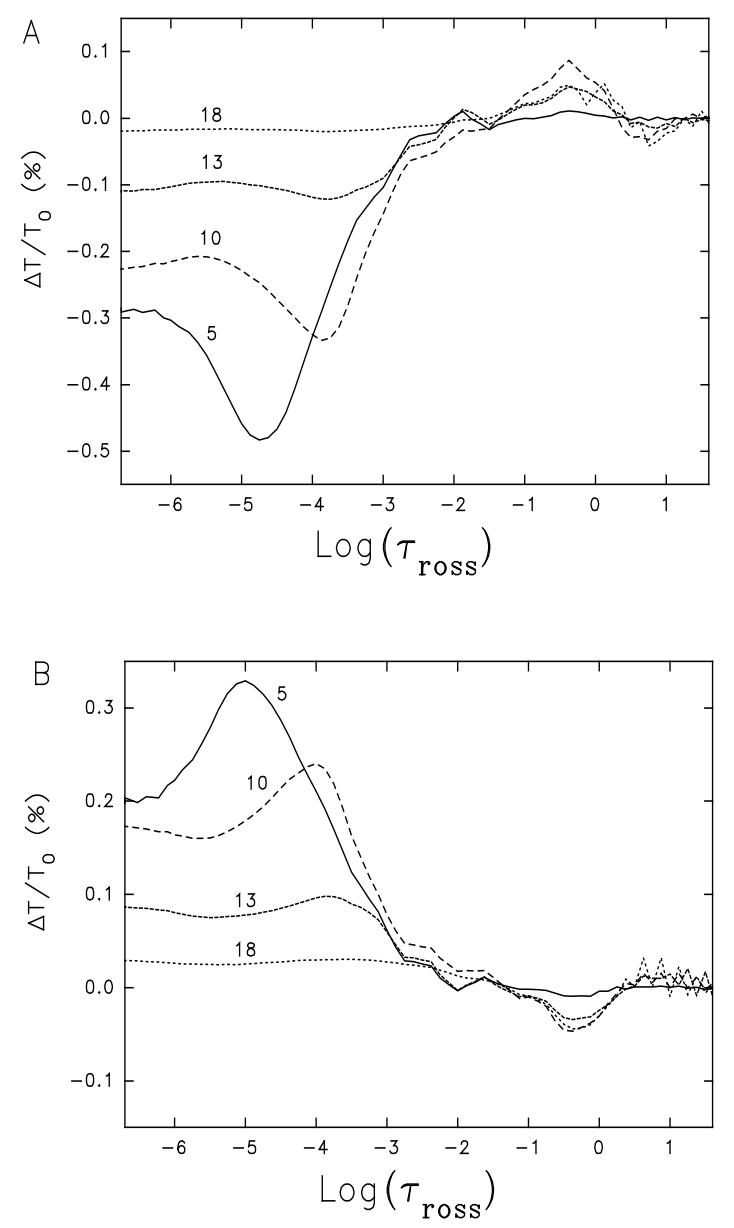

Fig. 4. The temperature perturbations in the magnetic models relative to the reference non-magnetic model. a) The outward-directed Lorentz force; $\mathbf{b})$ the inward-directed magnetic force.

atmospheric structure modified by the presence of non-forcefree magnetic fields. This is illustrated by the difference of synthetic $\mathrm{H} \beta$ profiles presented in Fig. 5. Hydrogen line profiles were computed with the BALMER code (Peterson 1969), modified to include the recent Stark broadening calculations by Stehlé (1994), instead of the Vidal et al. (1973) Stark broadening tables. The profiles obtained using the models with the outward-directed magnetic force correspond to standard hydrogen lines computed for models with lower surface gravity. The situation is opposite for the inward-directed Lorentz force. In this case hydrogen line profiles emergent from the magnetic atmosphere are deeper and correspond to a higher surface gravity in comparison with the results obtained for the non-magnetic model. However, the amplitude of this effect is smaller than for the outward-directed magnetic force.

Figure 6 shows the maximum difference between $\mathrm{H} \beta$ profiles derived from standard and magnetic atmospheres computed for various values of the local magnetic field strength and equatorial induced electric field $E_{\text {eq }}=5 \times 10^{-11}$. The wavelength location of the maximum difference between $\mathrm{H} \beta$ profiles depends slightly on the local (equatorial) magnetic field strength adopted for the model computation and occurs at around $10 \AA$ from the line core (see Fig. 5). The behaviour
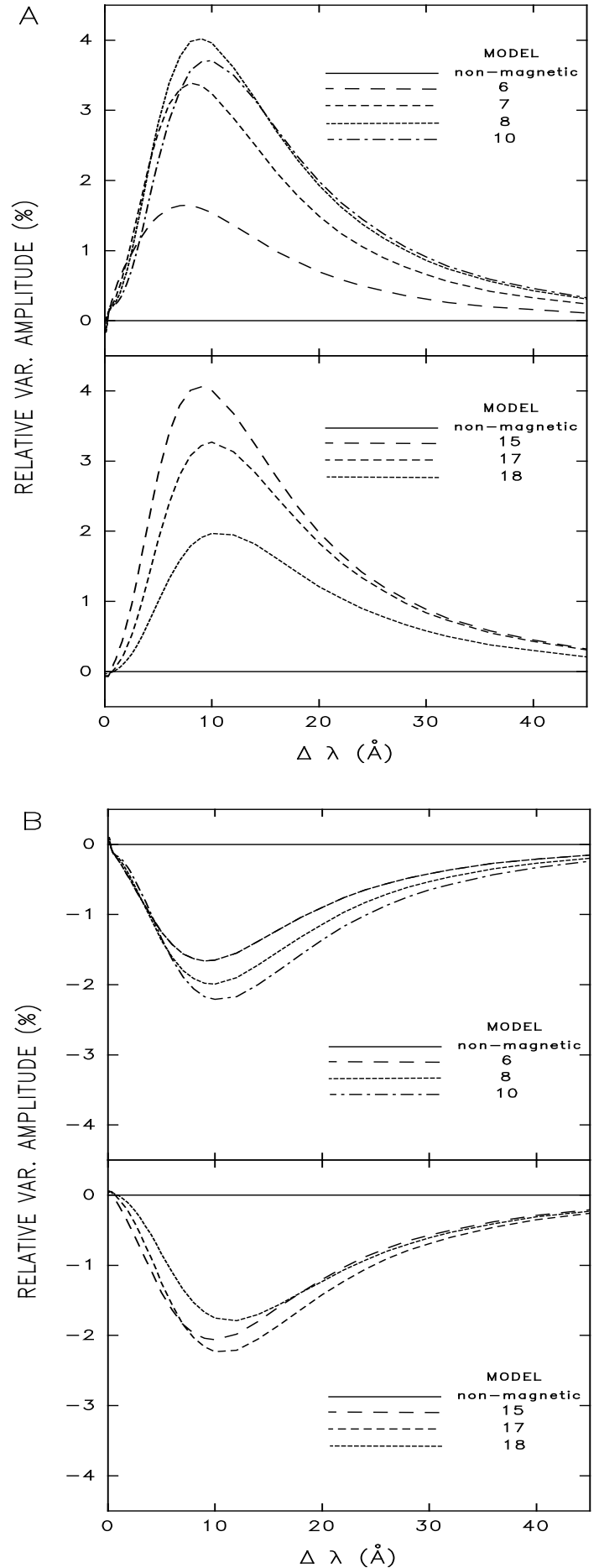

Fig. 5. The differences between the magnetic and non-magnetic $\mathrm{H} \beta$ profiles. a) The outward-directed Lorentz force; b) the inwarddirected magnetic force.

of the maximum difference as a function of magnetic field strength shows two extrema which are caused by the bimodal structure of the variation of effective gravity with depth in magnetic atmospheres. For very small magnetic fields the perturbation of the atmosphere is limited to small Rosseland optical depths above the region where the wings of Balmer lines form. Increasing the magnetic field strength leads to a shifting of the perturbation towards the deeper regions which contribute to the 


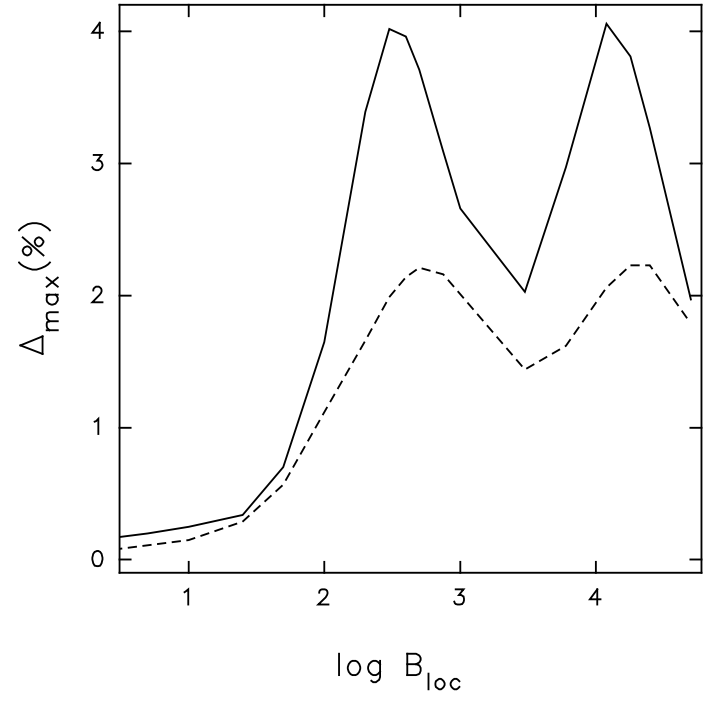

Fig. 6. The maximum difference between $\mathrm{H} \beta$ profiles computed for magnetic and non-magnetic atmospheres as a function of the magnetic field strength $B_{\text {loc }}$. The solid line illustrates the outward-directed Lorentz force, while the dashed line corresponds to the inwarddirected magnetic force.

formation of hydrogen lines. In this process two maxima of the effective gravity perturbation pass through the zone of Balmer line formation, giving rise to the bimodal structure in Fig. 6. A very strong magnetic field $(\gg 10 \mathrm{kG})$ considerably reduces the total electrical conductivity of all atmospheric layers which influence the formation of Balmer lines. As a result these layers are in an almost force-free state and no strong Balmer line anomaly occurs.

All results presented above illustrate the effect of the Lorentz force in the atmosphere of a magnetic star with an effective temperature of $11000 \mathrm{~K}$ and surface gravity $\log g=4.0$. It is interesting to examine the influence of a magnetic field in stars with different atmospheric parameters. We found that variation of the surface gravity within the range of $\log g$ typical for main sequence stars does not significantly modify the effects due to the magnetic field. Changes in stellar temperature are more important. Figure 7 shows the maximum difference between $\mathrm{H} \beta$ profiles computed for magnetic and non-magnetic models atmospheres with different $T_{\text {eff }}$ typical for CP stars, $B_{\text {loc }}=500 \mathrm{G}$ and $E_{\text {eq }}=5 \times 10^{-11}$. The behaviour of the maximum difference as a function of temperature is essentially defined by the ionization fraction of atmospheric plasma. For stars with effective temperatures below $\approx 10000 \mathrm{~K}$ the Lorentz force is decreased by the presence of significant fraction of neutral particles, insensitive to the magnetic fields. Thus, if the induced electric field is generated by some universal mechanism for all magnetic CP stars, we expect that the influence of electromagnetic forces on the atmospheric structure will be smaller for cooler stars.

\subsection{Spectral variations of rotating magnetic stars}

Calculations presented in the previous section illustrated the difference between the local magnetic and non-magnetic

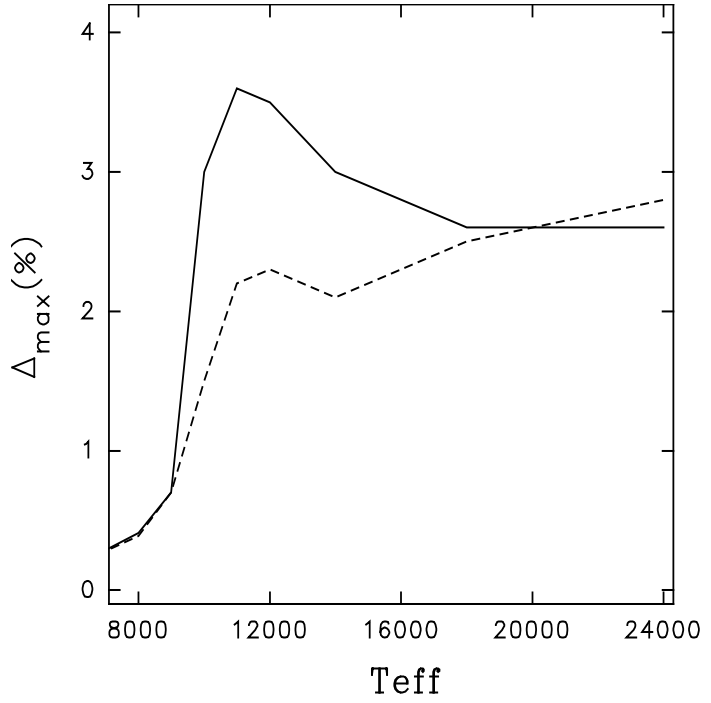

Fig. 7. The maximum difference between $\mathrm{H} \beta$ profiles computed for magnetic $\left(B_{\mathrm{loc}}=500 \mathrm{G}\right)$ and non-magnetic atmospheres as a function of the stellar effective temperature. The solid line illustrates the outward-directed Lorentz force, while the dashed line corresponds to the inward-directed magnetic force.

stellar model atmospheres. Such models essentially correspond to magnetic equatorial and polar regions of a real star. As one can see from Figs. 4 and 5, perturbation due to the Lorentz force can be quite significant at the magnetic equatorial plane and result in a strong anomaly of Balmer line profiles. However, in a real case of a rotating magnetic star, averaging over the stellar disk will reduce the net effect of the magnetic force. In this section we attempt to estimate the rotational variability of $\mathrm{H} \beta$ taking into account disk-averaging effects. As we explained in Sect. 5 in this exploratory study of Balmer line variability we do not carry out direct disk integration of the local line profiles or other observed stellar characteristics, but calculate disk integrated projection of the tangential field component onto the magnetic axis $\left\langle\sin \theta \boldsymbol{B}_{\theta}\right\rangle$ for each rotational phase and use it as an input parameter for model atmosphere calculation. Disk integration is performed using the expression

$$
\begin{aligned}
\left\langle\sin \theta B_{\theta}\right\rangle= & \frac{3}{\pi(3-u)} \int_{0}^{2 \pi} \mathrm{d} \varphi_{\star} \int_{0}^{\pi / 2} \mathrm{~d} \theta_{\star} \sin \theta_{\star} \cos \theta_{\star} \\
& \times\left(1-u+u \cos \theta_{\star}\right) \sin \theta B_{\theta},
\end{aligned}
$$

where $\varphi_{\star}$ and $\theta_{\star}$ are spherical coordinates in the stellar reference frame and the limb darkening coefficient $u=0.4$ as predicted for $\mathrm{H} \beta$ wavelength by the ATLAS9 atmosphere with $T_{\text {eff }}=11000 \mathrm{~K}$ and $\log g=4.0$. It is useful to compare variations of $\left\langle\sin \theta B_{\theta}\right\rangle$ with other traditional disk-integrated magnetic observables, such as field modulus $\langle B\rangle$ and longitudinal field $\left\langle B_{\mathrm{z}}\right\rangle$. Figure 8 shows an example of phase curves for these magnetic parameters computed for a dipolar magnetic field with $B_{\mathrm{p}}=1000 \mathrm{G}$ in the situation where the rotation axis is perpendicular to the magnetic axis and to the line of sight (i.e., inclination angle $i=90^{\circ}$ and magnetic obliquity $\beta=90^{\circ}$ ). In this and other magnetic dipolar configurations $\left\langle\sin \theta B_{\theta}\right\rangle$ is positive but is always lower than the equatorial magnetic field strength. 


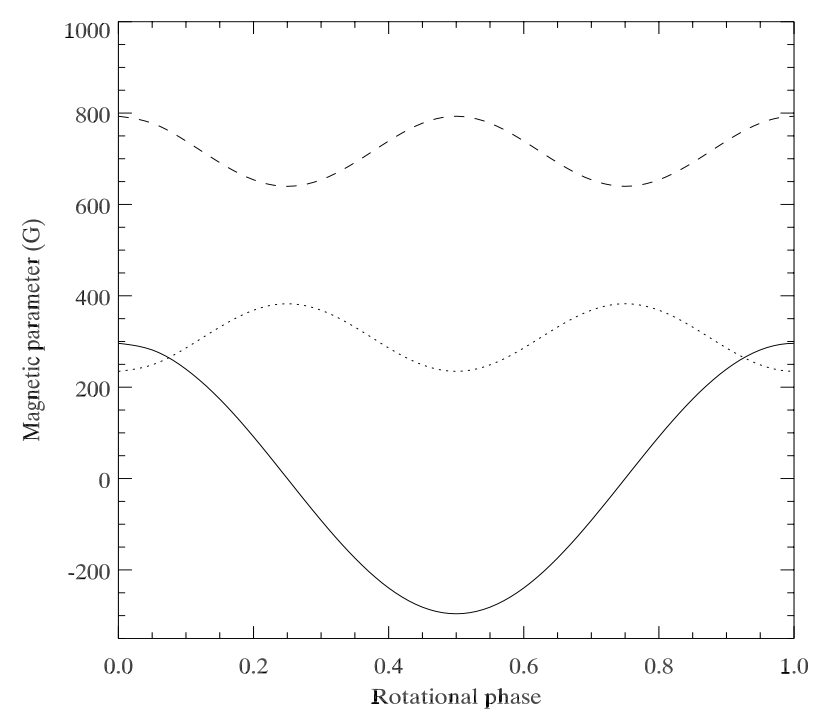

Fig. 8. Phase variation of the disk-integrated magnetic parameters for the dipolar field geometry with the polar strength $B_{\mathrm{p}}=1 \mathrm{kG}, \beta=90^{\circ}$ and $i=90^{\circ}$. The solid line shows the phase curve of the longitudinal field $\left\langle B_{z}\right\rangle$, dashed line corresponds to the field modulus $\langle B\rangle$ and dotted line - to $\left\langle\sin \theta B_{\theta}\right\rangle$.

Even in the case when a rotating star shows the observer both the magnetic equator (zero $\left\langle B_{\mathrm{z}}\right\rangle$ ) and magnetic polar regions (positive and negative extrema of $\left\langle B_{\mathrm{z}}\right\rangle$ ), the difference between the Balmer line profiles obtained at different rotational phases will be smaller than the difference between magnetic and nonmagnetic profiles discussed in Sect. 6.1. However, if the induced currents are large enough, variability of hydrogen lines can still be observed. This is illustrated in Fig. 9, where the differences between $\mathrm{H} \beta$ profiles are plotted as a function of distance from the line core. As one can see from the comparison of this figure and Fig. 5, for $1 \mathrm{kG}$ dipolar field the effect of disk averaging over the visible stellar hemisphere reduces the local differences between $\mathrm{H} \beta$ line profiles by roughly a factor of 3 .

The structure of the phase curves of the Balmer line variation may have a rather different appearance depending on the orientation of stellar rotational and magnetic axes. To illustrate this effect, Fig. 10 shows the relative equivalent width variation of the first three hydrogen Balmer lines for nine different combinations of the angles $i$ and $\beta$. We found that when both $i$ and $\beta$ are small, Balmer line variations will show one maximum and one minimum during the rotational cycle. Otherwise, when $i$ and/or $\beta$ is large, double-wave variation of hydrogen lines will be observed. For the most favourable magnetic geometry $\left(i=90^{\circ}\right.$ and $\left.\beta=90^{\circ}\right)$ and the induced electric field $E_{\mathrm{eq}}=5 \times 10^{-11}$ variability of the equivalent width of $\mathrm{H} \beta$ may reach up to $4 \%$. As expected from the assumptions of our model, the phase variation of the equivalent widths is symmetric with respect to phase 0.5 . Such a situation is characteristic of our model because the distribution of the Lorentz force over the stellar surface follows the symmetry relative to the axis of the magnetic dipole and does not depend on the sign of the disk-averaged longitudinal magnetic field.

Calculations presented in this section demonstrate that even comparatively weak magnetic fields can lead to significant
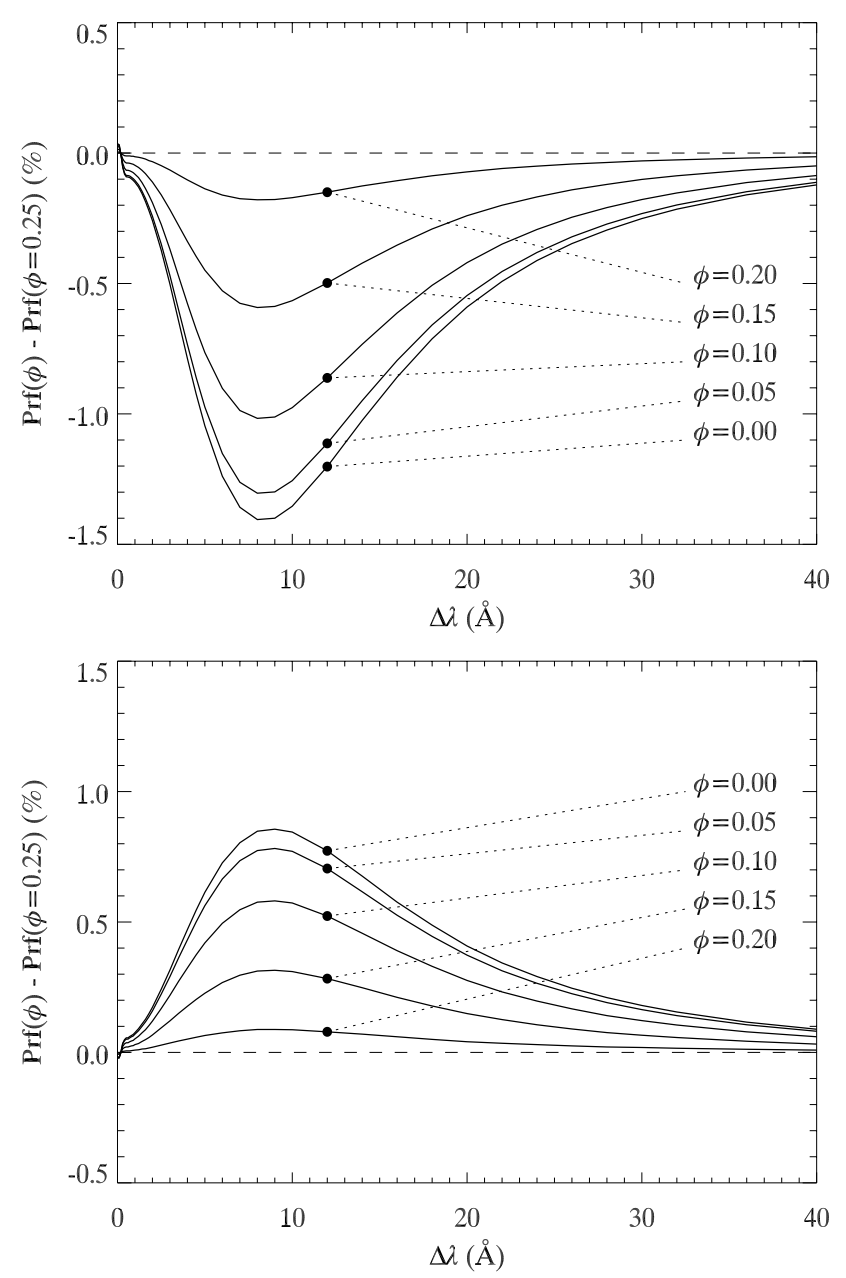

Fig. 9. Phase variation of the disk-integrated $\mathrm{H} \beta$ profiles for a rotating magnetic star with $1 \mathrm{kG}$ dipolar field and $E_{\mathrm{eq}}=5 \times 10^{-11}$. Hydrogen line profiles are plotted for phases $0.00,0.05,0.10,0.15$ and 0.20 relative to the profile corresponding to the rotational phase 0.25 (strongest magnetic field effect). The upper plot is for the outward-directed Lorentz force, while the lower plot illustrates results for the inwarddirected magnetic force.

variability of Balmer lines. Since distribution of the field strengths of typical early-type magnetic stars peak at around $1 \mathrm{kG}$ (Bohlender \& Landstreet 1990) and vary by a factor of a few, the amplitude of hydrogen line variations will primarily depend on the magnitude of the induced electric field. Several hypothetical processes which can give rise to such fields will be discussed in Sect. 8, but before that we will examine existing observational material on the periodic Balmer line variability in magnetic stars and try to interpret these data in the context of variability induced by the Lorentz force.

\section{Comparison with observations}

As we already mentioned in Sect. 2, there exists some observational evidence for the presence of a detectable Lorentz force in the atmospheres of magnetic CP stars. The spectroscopic data presented by Kroll (1989) demonstrated that some stars show variability of Balmer line profiles of up to $5 \%$. In 

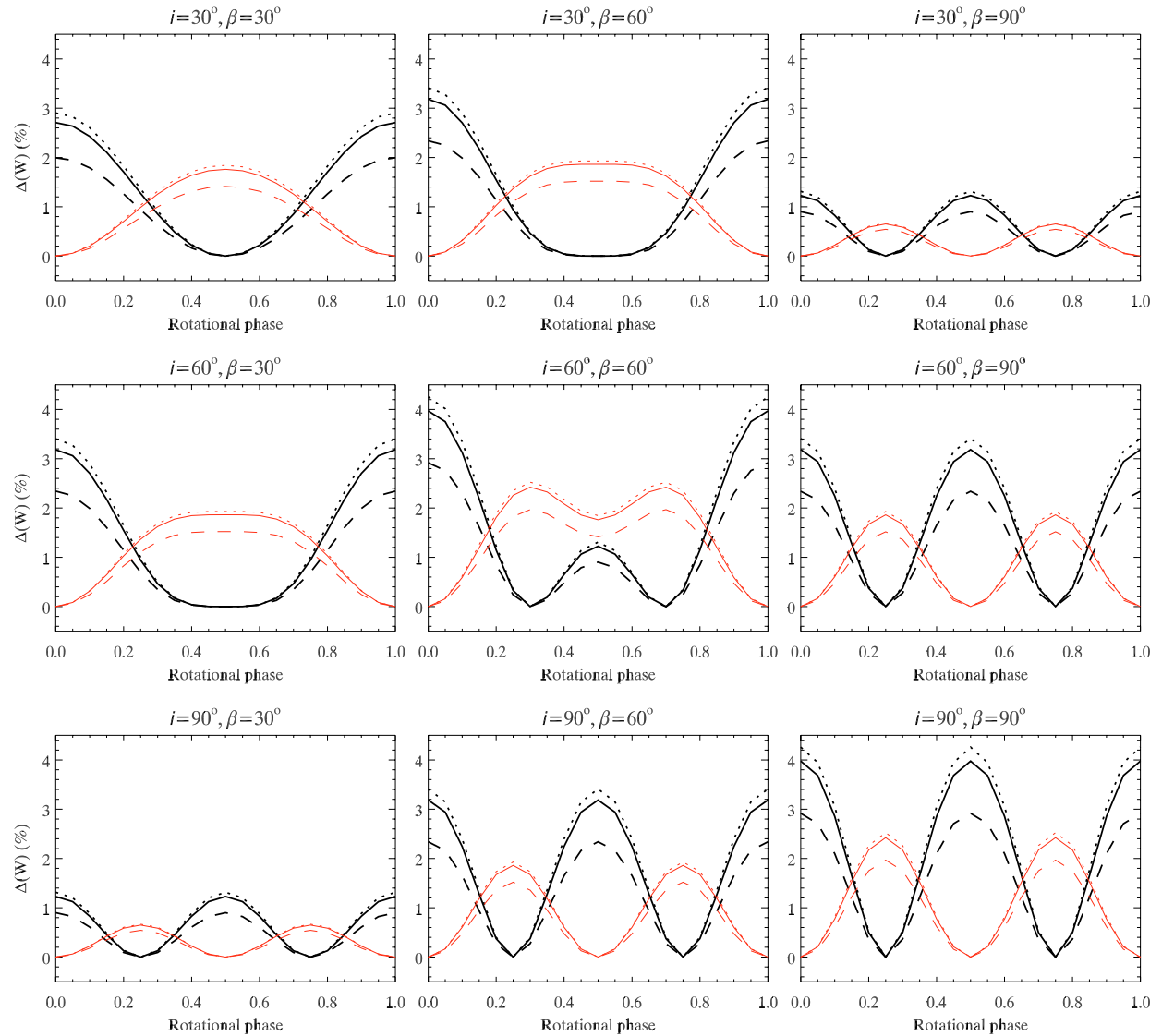

Fig. 10. Phase variation of the equivalent width of hydrogen Balmer lines for different stellar inclinations $i$ and magnetic obliquities $\beta$ of the $1 \mathrm{kG}$ dipolar field and $E_{\mathrm{eq}}=5 \times 10^{-11}$. The thick curves correspond to the outwarddirected Lorentz force, while the thin lines show calculations for the inwarddirected magnetic force. Dashed lines show variation of $\mathrm{H} \alpha$, solid lines $-\mathrm{H} \beta$ and dotted lines $-\mathrm{H} \gamma$. many cases, the wavelength dependence of the variability amplitude is in good agreement with the predictions of our models. The CP star 56 Ari provides the best example. In this star the amplitude of the $\mathrm{H} \beta$ profile variability reaches $2 \%$, with a well-defined maximum at about 6-10 $\AA$ from the line center, whereas in the core of $\mathrm{H} \beta$ the amplitude drops to zero, exactly as in our Fig. 9. Varying various parameters of standard nonmagnetic model atmospheres, Kroll (1989) suggested that such a shape can be produced only by variations of effective gravity over the stellar surface. Assuming that the variability of hydrogen lines in 56 Ari is related to the evolution of an approximately dipolar magnetic field with parameters determined by Borra \& Landstreet (1980), we can derive the induced equatorial electric field required to reproduce the observed variability amplitudes. Using the modelling approach outlined in the previous section, we found $E_{\mathrm{eq}}=8 \times 10^{-11}$ for the case of the outward-directed Lorentz force and $E_{\mathrm{eq}}=4 \times 10^{-10}$ for the scenario with the inward-directed magnetic force.

Unfortunately, apart from the study by Kroll (1989), who presented observations of rotational modulation of Balmer lines in only a few CP stars, there have been no other systematic spectroscopic surveys of Balmer line variability. On the other hand, one can make use of $\mathrm{H} \beta$ photometric observations, published for a large number of magnetic CP stars. Although $\mathrm{H} \beta$ photometry is not informative enough to permit analysis of rotational modulation of hydrogen line profiles, it allows reliable detection of the variation of the equivalent width of the $\mathrm{H} \beta$ line.
The observational aspect of the problem of $\beta$-index variability was examined in detail by Musielok \& Madej (1988). In this paper the authors presented an extensive set of $\mathrm{H} \beta$ photometric measurements for $22 \mathrm{CP}$ stars. A large fraction of these objects show both equatorial and polar magnetic regions during their rotation. In addition, some of the stars from the sample of Musielok \& Madej show variation of the longitudinal field, which is almost symmetric with respect to zero, indicating large inclination angles. As we demonstrated above (see Fig. 10), such stars are expected to show a bimodal structure of the phase variation of the equivalent width (or $\beta$-index) of Balmer lines. Indeed, there are examples of such a behaviour in the $\mathrm{H} \beta$ data collected by Musielok \& Madej. However, more stars do not show such a clear bimodal structure in the rotational modulation of the $\beta$-index and have only one minimum and one maximum per rotation cycle. This lack of stars with the behaviour expected for simple dipolar fields may be related to the modification of atmospheric currents by the processes neglected in our models (e.g. Hall drifts, meridional circulation, ambipolar diffusion) or reflect departures from the pure axisymmetric dipolar magnetic topology examined here. On the other hand, in certain situations we can expect that an inhomogeneous distribution of chemical abundances over the stellar surface may produce an important contribution to the variation of the $\beta$-index due to changes of metallic lines within the filter bandpasses. All these scenarios could be distinguished by using high-resolution spectroscopic observations instead of $\mathrm{H} \beta$ photometry. 
Faced with the scarcity and ambiguity of the observational data available from the literature, in this paper we will not discuss variations of Balmer lines in individual CP stars. The aim of our preliminary attempt to compare predictions of theoretical models with observations is to explain only the most general tendencies in the observed variability of the $\beta$-index given all our modelling assumptions. According to Musielok \& Madej (1988), the typical value of the amplitude of the $\beta$-index variation is roughly $0.02 \mathrm{mag}$. Supposing that this variation of hydrogen lines is due to the Lorentz force induced by the evolution of the global dipolar magnetic fields, we found that, within the framework of our model, the value of $E_{\mathrm{eq}}$ required to produce such variations is of the order of $10^{-10} \mathrm{~cm}^{-1 / 2} \mathrm{~g}^{1 / 2} \mathrm{~s}^{-1}$ for both directions of the magnetic field evolution (decay and generation).

\section{Discussion and conclusions}

We briefly summarize main assumptions and findings of our study. In our model the influence of a magnetic field on the atmospheric structure is taken into account by including the Lorentz force in the equation of magnetohydrostatic equilibrium. The Lorentz force term originates due to a slow evolution of the global magnetic field with nearly dipolar surface geometry. The distribution of the magnetic force over the stellar surface corresponds to the latitudinal distribution of the induced electric current with a maximal impact on the atmospheric structure at the magnetic equator and no influence at the magnetic poles. For a given strength of the surface magnetic field, the amplitude of the atmospheric perturbation in our model depends on the strength and the sign of the induced electric field. We carried out detailed modelling of the local interaction between the induced electric currents and magnetized stellar plasma and obtained the following results for representative values of the strength of the induced electric field:

1. The behaviour of the effective gravity with the vertical direction in the stellar atmosphere has two extrema, which are formed by the electrical conductivity by electrons (in the deeper atmospheric layers) and by ions (in the upper layers). This result suggests that the inclusion of the ion component in any calculations of the magnetized atmosphere is important, especially for strongly magnetic stars for which the ion component of the induced currents dominates in all atmospheric layers (see Fig. 2).

2. For any value of the surface field strength in the range typically found in magnetic CP stars $\left(10^{2}-10^{4} \mathrm{G}\right)$, substantial distortions in the stellar atmospheric structure occur if the equatorial strength of the induced electric field exceeds approximately $10^{-11} \mathrm{~cm}^{-1 / 2} \mathrm{~g}^{1 / 2} \mathrm{~s}^{-1}$. Generally, the inward-directed Lorentz force produces smaller perturbations of the atmospheric structure compared with the outward-directed magnetic force with the same amplitude.

3. The maximal perturbation of the Balmer line shapes due to the Lorentz force is expected to occur within the two ranges of the surface magnetic field in CP stars: from about $500 \mathrm{G}$ to $1 \mathrm{kG}$ and for fields $\geq 8 \mathrm{kG}$ (see Fig. 6).
4. Typical amplitudes of the variation of $\mathrm{H} \beta$ index in $\mathrm{CP}$ stars can be ascribed to the presence of a non-zero Lorentz force if the induced electric field in stellar atmospheres reaches the value of $\sim 10^{-10} \mathrm{~cm}^{-1 / 2} \mathrm{~g}^{1 / 2} \mathrm{~s}^{-1}$.

These results were obtained under the assumption that the equatorial induced electric field is constant in all atmospheric layers. This should be close to reality if we deal with an evolving global magnetic field for which changes in strength throughout the observable atmospheric layers are very small and can be disregarded. This assumption, however, may not be applied if the Lorentz force has a different nature, for example, produced by ambipolar diffusion. Nevertheless, our results provide an observational test to distinguish these effects. As was shown by LeBlanc et al. (1994), the ambipolar diffusion is small for the weak field stars and increases in stars with the surface magnetic fields of about $10 \mathrm{kG}$ and higher. It should be noted, however, that many weak field magnetic CP stars discussed by Musielok \& Madej (1988) exhibit extremely high amplitudes of $\mathrm{H} \beta$ profile variation and this cannot be fully explained by the ambipolar diffusion.

Clearly, the most promising direction of the study of the possible origin of the Lorentz force in the atmosphere of CP stars would be an analysis of the effects related to the global field evolution. Any temporal variation of a magnetic field in stellar atmospheres leads to development of an induced electric field and the corresponding current of charged particles in all conductive atmospheric layers:

$\nabla \times \boldsymbol{E}=-\frac{1}{c} \frac{\partial \boldsymbol{B}}{\partial t}$

Therefore, direct observations of the induced electrical field $\boldsymbol{E}$ may provide an indicator of the processes governing the field evolution and determining typical strength of the dipole component of a global field and the characteristic time scale of its evolution. In the next two sections we attempt to make a connection between the induced electrical field and the possible mechanisms of field evolution. We use a simple schematic model which does not claim to provide a detailed description of the physics of CP stars but is nevertheless sufficient for a crude order of magnitude estimate.

\subsection{Decay of dipolar magnetic fields}

We follow Landstreet (1987) in estimating the strength of the electric field induced at the stellar surface by a decay of a quasidipolar fossil magnetic field. In this idealized model, the magnetic field has a dipolar geometry throughout the magnetic star. In this situation, the electric field induced at the equator can be approximated by the following expression:

$E_{\mathrm{eq}} \sim \frac{R_{\star} B_{\mathrm{eq}}}{c t}$,

where $R_{\star}$ is the stellar radius, $B_{\text {eq }}$ is the strength of the dipolelike surface field at the magnetic equator and $t$ is the characteristic decay time of the magnetic field. For a typical A0 magnetic star with a surface field of $10 \mathrm{kG}$ this leads to the value $E_{\text {eq }} \sim 10^{-13} \mathrm{~cm}^{-1 / 2} \mathrm{~g}^{1 / 2} \mathrm{~s}^{-1}$, which is $2-3$ orders of magnitude 
smaller than the strength of the electric currents tested in our model calculations and inferred from the variation of hydrogen lines. The former theoretical estimate of $E_{\text {eq }}$ is based on the $10^{10} \mathrm{yr}$ typical time for the decay of nearly dipolar fields in middle main-sequence stars (Moss 1984; Cowling 1945). Clearly, such a slow evolution of the global field has a negligible effect on the atmospheric structure and cannot produce observable variations in the Balmer line profiles. On the other hand, the decay time of $10^{7}-10^{8} \mathrm{yr}$ corresponding to the values of $E_{\text {eq }}$ tested throughout our paper (necessary to produce observable changes in stellar atmospheric structure) and tentatively derived from variations of hydrogen lines (Sect. 7) is much shorter than the classical value obtained by Moss (1984) and other authors under a set of simplifying assumptions.

Thus, if observations of the strong electric currents in atmospheres of CP stars are confirmed, we may conclude that the general concept of a slow decay of fossil fields in magnetic CP stars is invalid. The large amplitude of the induced electric currents may indicate that the dipolar geometry, dominating at the surface, becomes significantly distorted inside a magnetic star. Such a distortion is very likely to occur due to the existence of a convective zone or other dynamical processes in subphotospheric stellar layers. Alternatively, even in the absence of processes of dipolar field distortion inside a magnetic CP star, motions of stellar plasma induced by stellar rotation or large-scale diffusion currents (like ambipolar diffusion) may also lead to a significant surface Lorentz force.

We hope that our analysis will stimulate new attempts of detailed MHD modelling of $\mathrm{CP}$ stars with alternative boundary conditions. By taking into account observed strength of the induced surface electric field as an additional boundary condition, estimates of the decay time and the characteristic strength and configuration of the interior magnetic field can be studied in a much more detailed and reliable way.

\subsection{Generation of global magnetic fields}

The most recent statistical study of ages of magnetic CP stars (Hubrig et al. 2000) revealed an interesting aspect directly related to the problem of magnetic field origin and evolution. The authors found that there are no young magnetic stars among cooler CP stars. This clearly contradicts the hypothesis that global stellar magnetic fields decay within the main sequence lifetime. The absence of magnetic stars close to the ZAMS may indicate that the generation of strong fields by some hypothetical dynamo processes is connected with a specific phase of main sequence stellar evolution. Another interpretation is that magnetic field, initially buried during the stellar formation phase, diffuses outward as the star ages. Some of these possible scenarios of emergence of magnetic fields in photospheres of main sequence stars were discussed by Moss (2001).

Alternatively, the apparent relation between stellar ages and the incidence of magnetism may be due to an observational bias: young strongly magnetic stars may have not been detected because of the reasons mentioned by Hubrig et al. (2000). This view is supported by the recent discovery of a young lowmass magnetic CP star (Bagnulo et al. 2003). Thus, from the observational point of view, the conclusions of Hubrig et al. certainly require verification with a study of a larger sample of magnetic CP stars. However, it would also be very interesting to examine the theoretically possible physics of magnetic field generation.

Within the framework of our modelling approach the sign of the Lorentz force can be uniquely determined from the variation of hydrogen lines and can be used to find a general direction of the surface field evolution. As follows from the expressions discussed by Wrubel (1952), the surface Lorentz force is likely to be inward-directed in the situation where the field decays in the process of stellar evolution on the main sequence. The opposite sign of the Lorentz force may be indicative of the field generation processes.

\subsection{Final remarks}

There are many ways to create a Lorentz force in stellar atmospheres. This includes deviation of the magnetic field from a poloidal field geometry (for example the distorted dipolar topology as discussed by Stępień 1978), ambipolar diffusion (LeBlanc et al. 1994), rotationally induced meridional circulation in magnetic stars (Moss 1984), interaction between the radiatively driven flow of charged particles and the magnetic field (Peterson \& Theys 1981), evolution of the global magnetic field, etc. Thus, we do not claim that our consideration is the only way to address the problem of the generation of the Lorentz force in the atmosphere of CP stars. The problem is too complex, and comprehensive answers can be obtained only within the framework of a self-consistent MHD study. Magnetic field dynamics, effects of ambipolar diffusion, chemical inhomogeneities and other phenomena need to be studied simultaneously and self-consistently and eventually related to the analysis of high-resolution spectroscopic material. Nevertheless, as follows from the analysis presented in this paper, our consideration, which starts with the assumption of electric currents, is one of the most general ways of dealing with the problem. It allows us to continue modelling the Lorentz force in the context of global magnetic field evolution. As the next step, it is necessary to develop a more realistic model of rotating stars including Hall's currents, ambipolar diffusion and some other effects that may produce a variation of the effective gravity. It would also be useful to extend the spectral predictions of our models to other absorption lines in addition to lines of neutral hydrogen.

In addition to theoretical modelling, the problem of magnetic field evolution can be resolved with the help of an unbiased statistical study. Obtaining frequencies of occurrence of magnetic stars during their evolution on the main sequence is an extremely important supplementary method that can help to constrain uncertain parameters of theoretical models. Such a statistical method was developed and applied to magnetic white dwarfs (Valyavin \& Fabrika 1999). Intriguingly, the situation of global magnetic fields of white dwarfs is reminiscent of the results of the study by Hubrig et al. (2000): white dwarfs with strong fields show a tendency for the magnetic field to increase with the age of the stellar remnant (Valyavin \& Fabrika 1999; Liebert et al. 2003). Unfortunately, the situation for the 
statistics of magnetic main sequence stars is unclear due to several selection effects. Apart from the suggestive results of Hubrig et al., no definite conclusions can be reached so far.

Acknowledgements. We wish to thank Dr. V. Tsymbal and Prof. S. Fabrika for useful discussions and Dr. I. Romanyuk and Prof. Yu. Glagolevskij for critical advice and providing some of their results.. The help of Dr. P. Barklem, whose comments improved the text of the manuscript, was greatly appreciated. We are especially grateful to Prof. John Landstreet for helpful discussions, valuable suggestions and his interest in our work. We also sincerely thank the referee, Dr. G. Wade, whose constructive remarks contributed to the improvement of this paper. G.V. acknowledges Swedish Royal Academy of Sciences for support of his travel to Sweden and the Russian Academy of Sciences for partial financial support (RFBR grant N 01-02-16808). This work was also partially supported by the Lise Meitner fellowship to OK (FWF project M757-N02).

\section{Appendix: Electrical conductivity of stellar plasma}

For low-density plasma in the absence of a magnetic field the following expressions can be used to calculate the mean freepath time $\tau_{i}$ and electrical conductivity $\lambda_{i}$ :

$\tau_{i}=l_{i} /\left\langle v_{i}\right\rangle$,

where $\left\langle v_{i}\right\rangle$ is the root mean square velocity $\left(\sqrt{3 k T / m_{i}}\right), l_{i}$ is the mean free-path length and index $i$ runs through all types of particles. In the general case:

$l_{i}^{-1}=\sum_{k} l_{i k}^{-1}$,

where $l_{i k}$ is the mean free-path length of the $i$-type particles interaction with the $k$-type particles.

Methods for calculation of $l_{i k}$ are derived from the kinetic theory of gases. In this paper we use the solution given by Pikelner (1966):

$l_{\mathrm{ee}}=\frac{(k T)^{2}}{n_{\mathrm{e}} e^{4} L_{\mathrm{ee}}}, \quad l_{i k}=\frac{(k T)^{2}}{n_{k} e^{4} L_{i k} Z_{\mathrm{i}}^{2} Z_{k}^{2}}, \quad l_{\mathrm{e} k}=\frac{(k T)^{2}}{n_{k} e^{4} L_{\mathrm{e} k} Z_{k}^{2}}$,

where subscript "e" corresponds to the electronic gas component, $Z_{\mathrm{i}}$ is the ionization degree (unity for the electrons) and auxiliary quantities $L_{i k}$ are defined with

$L_{i k}=2 \ln \frac{3(k T)^{3 / 2}}{2 \pi^{1 / 2} n_{k}^{1 / 2} e^{3} Z_{\mathrm{i}} Z_{k}}$.

The mean free-path length $l_{i a}$ of particles of type $i$ interaction with $a$-type neutral atoms is given by:

$l_{i a}=\frac{1}{\sigma_{a} n_{a}}$,

where $\sigma_{a}$ is the effective cross-section of neutral atoms of type $a$.
Finally, electrical conductivity of the $i$-type plasma fraction is obtained from

$\lambda_{i}=\frac{n_{i} e_{i}^{2} \tau_{i}}{m_{i}}$

\section{References}

Bagnulo, S., Landi Degl'Innocenti, M., Landolfi, M., \& Mathys, G. 2002, A\&A, 394, 1023

Bagnulo, S., Landstreet, J. D., Lo Curto, G., Szeifert, T., \& Wade, G. A. 2003, A\&A, 403, 645

Bohlender, D. A., \& Landstreet, J. D. 1990, MNRAS, 247, 606

Borra, E. F., \& Landstreet, J. D. 1980, ApJS, 42, 421

Borra, E. F., Landstreet, J. D., \& Mestel, L. 1982, ARA\&A, 20, 191

Carpenter, K. G. 1985, ApJ, 289, 660

Cowling, T. G. 1945, MNRAS, 105, 166

Cowling, T. G. 1957, Magnetohydrodynamics, London

Glagolevskij, Yu. V., \& Chountonov, G. A. 2001, Bull. Spec. Astrophys. Obs., 51, 88

Hubrig, S., North, P., \& Mathys, G. 2000, ApJ, 539, 352

Kochukhov, O., Piskunov, N., Ilyin, I., Ilyina, S., \& Tuominen, I. 2002, A\&A, 389, 420

Kochukhov, O., Bagnulo, S., Wade, G. A., et al. 2004, A\&A, 414, 613

Kroll, R. 1989, Rev. Mex. Astron. Astrofis., 2, 194

Kurucz, R. 1993, CD-ROM No. 13, Smithsonian Astrophys. Obs.

Landstreet, J. D. 1987, MNRAS, 225, 437

Landstreet, J. D. 2001, in Magnetic Fields Across HertzsprungRussell Diagram, ed. G. Mathys, S. K. Solanki, \& D. T. Wickramasinghe, ASP Conf. Ser., 248, 277

LeBlanc, F., Michaud, G., \& Babel, J. 1994, ApJ, 431, 388

Leibert, J., Bergeron, P., \& Holberg, J. B. 2003, AJ, 125, 348

Madej, J. 1981, Bull. Spec. Astrophys. Obs., 32, 24

Madej, J. 1983, Acta Astron., 33, 1

Madej, J. 1983, Acta Astron., 33, 253

Madej, J., Jahn, K., \& Stępień, K. 1984, Acta Astron., 34, 419

Moss, D. 1984, MNRAS, 207, 107

Moss, D. 2001, in Magnetic Fields Across Hertzsprung-Russell Diagram, ed. G. Mathys, S. K. Solanki, \& D. T. Wickramasinghe, ASP Conf. Ser., 248, 305

Musielok, B., \& Madej, J. 1988, A\&A, 202, 143

Parker, E. N. 1979, Cosmical magnetic fields (Oxford: Clarendon press)

Peterson, D. M. 1969, Smithsonian Astrophys. Obs. Spec. Rep., 293

Peterson, D. M., \& Theys, J. C. 1981, ApJ, 244, 947

Pikelner, S. B. 1966, Principles of cosmic electrodynamics, in Russian (Moscow: Nauka)

Schluter, A. 1950, Zs. f. Naturforsch, 5a, 72

Stehlé, C. 1994, A\&AS, 104, 509

Stępień, K. 1978, A\&A, 70, 509

Valyavin, G., \& Fabrika, S. 1999, in 11th European Workshop on White Dwarfs, ed. J.-E. Solheim, \& E. G. Meistas, ASP Conf. Ser., 169, 206

Vidal, C. R., Cooper, J., \& Smith, E. W. 1973, ApJS, 25, 37

Wrubel, M. H. 1952, ApJ, 116, 291 